This is the final peer-reviewed accepted manuscript of:

Falfari, S., Bianchi, G., Micci, G., Della Torre, A. et al., "Geometric and Fluid-Dynamic Characterization of Actual Open Cell Foam Samples by a Novel Imaging Analysis Based Algorithm" SAE Int. J. Engines 10(5):2275-2287, 2017

The final published version is available online at: https://doi.org/10.4271/2017-019288

Rights / License:

The terms and conditions for the reuse of this version of the manuscript are specified in the publishing policy. For all terms of use and more information see the publisher's website. 


\title{
GEOMETRIC AND FLUID-DYNAMIC CHARACTERIZATION OF Actual Open Cell FoAm Samples by a Novel Imaging ANALYSIS BASED ALGORITHM
}

A PREPRINT OF DOI:10.4271/2017-01-9288

\author{
Stefania Falfari, Gian Marco Bianchi, Giacomo Micci \\ University of Bologna
}

Augusto Della Torre, Gianluca Montenegro, Angelo Onorati

Politecnico di Milano

\author{
Sergio Negro \\ University of Toronto
}

2017

\section{Abstract}

Metallic open-cell foams have proven to be valuable for many engineering applications. Their success is mainly related to mechanical strength, low density, high specific surface, good thermal exchange, low flow resistance and sound absorption properties.

The present work aims to investigate three principal aspects of real foams: the geometrical characterization, the flow regime characterization, the effects of the pore size and the porosity on the pressure drop. The first aspect is very important, since the geometrical properties depend on other parameters, such as porosity, cell/pore size and specific surface. A statistical evaluation of the cell size of a foam sample is necessary to define both its geometrical characteristics and the flow pattern at a given input velocity. To this purpose, a procedure which statistically computes the number of cells and pores with a given size has been implemented in order to obtain the diameter distribution. In particular, a morphological characterization of an actual foam sample was performed by applying an image processing method. The analyzed foam was a cubic open-cell foam made by Silicon Carbide (SiC). Secondly, the pressure drop characterization of the foam sample was performed by means of CFD simulations. In particular, the relative importance of the viscous term and the inertial term was assessed. This analysis was performed including the effect of the porosity and of the mean pore diameter. The pressure gradient was parametrized with respect to such properties, passing through the concept of porosity and morphology. Additionally, the role of turbulence modeling in the simulation of the flow through the foam sample was investigated, finding that the flow pattern is not necessarily laminar and the turbulence is related to the fluid inertia.

\section{Introduction}

Recently, application of open-cell foams as substrates for exhaust after treatment devices of internal combustion engines has been an active area of research [1, 2, 3]. In particular, for this application open-cell foams are regarded with interest as a substitute for the traditional honeycomb monolithic substrate: the most attractive benefits are related to a better use of the catalytic surface due to the enhanced mass transport and to the quick heat up during engine cold starts. The pressure drop given by the foam is one of the key parameters in the design of devices involving such a porous material. Many works are available in the open literature investigating the phenomena leading to the pressure drop. They focus, in particular, on the influence of the flow regime and on the effects of the geometrical properties. With regard to the flow regime many authors $[4,5,6]$ agree that, in generic porous media, the transition from laminar to turbulent flow occurs at a critical Re number. The flow regime established inside the media for a particular pressure gradient is strictly dependent on the geometrical parameters, namely the porosity and the pore size. To characterize the geometry of a foam sample it is necessary to determine the quantities defining its micro-structure, such as the porosity $\epsilon$, the specific surface $S_{V}$, the cell size $d_{c}$ and the pore size $d_{p}$. With regard to an open-cell foam, the pore size deter- 


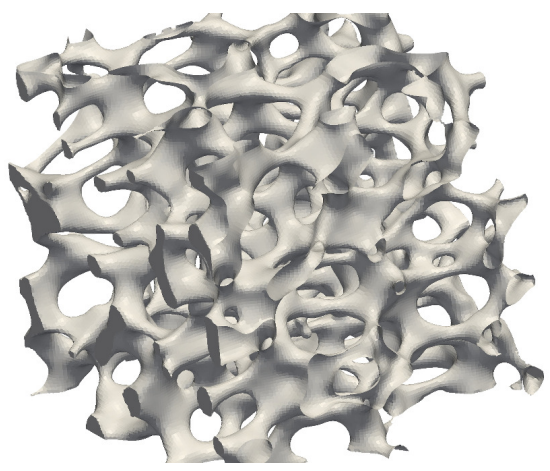

Figure 1: 3D surface reconstruction of the micro-structure of a foam sample.

mines the average dimension of the pore. It is expressed in terms of pore density (pore per inch, ppi), intending with this definition the number of pores that would subtend one linear inch. The cell size expresses the average dimension of the cell of the foam. Moreover the porosity of the foam is defined as the ratio between the volume of the fluid phase $V_{f}$ and the total volume of the porous medium $V$. The specific surface is defined as the ratio between the surface describing the fluid to solid interface $S$ and the total volume of the porous medium $V$.

Having set the properties of the foam sample, the authors [7] applied a genetic algorithm in order to address the design of catalytic devices equipped by metallic open-cell foam: the input parameters of the optimization process were the intrinsic characteristics of the foam and the external geometric dimensions of the catalytic support. The output parameters process were the pressure drop, the conversion efficiency and the cost effectiveness, related to the specific surface. The authors compared the foam struts with the standard honeycombs finding that the foams can achieve the same efficiency with about $1 / 4$ of the surface needed by the honeycombs.

In the present work, the main focus was to globally characterize the actual geometry of the foam sample, following an image based approach. To this purpose a sample of real silicon carbide ( $\mathrm{SiC}$ ), open-cell foam has been considered. Usually, the real foam sample micro-structure is reconstructed by means of computer tomography. Then, the cell and pore sizes of the actual foam sample are determined resorting to specific algorithms [8]. The approach proposed by the authors in the present work is based on an algorithm used in the image processing field. The starting image could come from a micro-tomography or from a reconstruction of an actual foam, as done by Lucci et al. [9], who created a structure based on randomly distributed Kelvin cells. On the other hand, the main disadvantage of this technique, based on the image analysis, is its limited accuracy, if compared to the one based on the micro-tomography, as it will be shown in the following paragraphs.

In this paper, foam sample geometry is characterized using an image processing technique. In a second phase, the effect of the porosity and of the mean pore diameter on the pressure gradient was assessed. To this purpose, the geometry was artificially modified by applying scaling or surface offset transformations to modify, in an independent way, parameters such as porosity or pore size [10]. Finally the turbulence effect on the foam sample was deeply analyzed by means RANS CFD simulations.

\section{Geometric analysis}

The geometrical characterization of the sample foam, i.e. the statistical evaluation of the cell and pore size, was necessary to determine more correctly the flow regime at a given inlet flow rate. A procedure allowing the statistical computation of the number of cells and pores of a given size has been implemented in order to obtain a distribution of diameters. The analysed foam sample was an open-cell foam made by silicon carbide SiC (Figure 1). Usually an actual foam sample is converted in an image in stl format by the application of the micro-tomography technique.

The focus of this first part of the work was to demonstrate how it is possible to characterize the foam structure (deriving mean cell and pore sizes) using a simple two-dimensional (2-D) or three-dimensional (3-D) image, not necessarily in stl format. Following this approach, the micro-tomography of the actual sample is no more strictly necessary.

Geometrical and thermophysical properties of the foam sample are listed in Table 1, where $\epsilon$ stands for porosity, $S_{V}$ for specific surface, $V$ for total volume, $\rho$ for material density, $\lambda$ for conductivity and $c_{S}$ for specific heat.

The foam properties are primarily determined by their structure: the various production processes configure different morphologies and geometries. Many studies have focused on techniques of representation and analysis of structures 
Foam sample main parameters

$\begin{array}{ll}\text { Length }[\mathrm{mm}] & 15.0 \\ \text { Width }[\mathrm{mm}] & 15.0 \\ \text { Height }[\mathrm{mm}] & 15.0 \\ \epsilon[\%] & 82.6 \\ d_{p}[\mathrm{ppi}] & 10 \\ S_{V}\left[\mathrm{~m}^{2} / \mathrm{m}^{3}\right] & 578.6 \\ V\left[\mathrm{~m}^{3}\right] & 3375 \cdot 10^{-9} \\ \rho\left[\mathrm{kg} / \mathrm{m}^{3}\right] & 3100 \\ \lambda\left[\mathrm{W} / \mathrm{mK}^{-}\right] & 270 \\ c_{S}[\mathrm{~J} / \mathrm{kgK}] & 750\end{array}$

Table 1: Open-cell foam sample geometrical and thermophysical parameters.

with elaboration of models suitable for defining the main characteristics proper to each foam. From a literature survey it appears that the most common models used for both metallic and ceramic foams are:

- The cubic-cell model [11], which is very simple but with obvious limits in the representation of the real structure;

- The model based on the tetrakaidecahedral cell, used for ceramic foams by Xu et al. [12] and for aluminium foams by Mancin et al. [13];

- The model based on the Kelvin cells randomly generated by Lucci et al. [9, 14].

Through these models it is possible to estimate the geometric characteristics of the foam strut and accordingly their fluid-dynamic characteristics. Results are generalized in terms of dimensionless numbers such as Reynolds number Re (flow regime), Sherwood number Sh (mass transfer) and Nusselt number Nu (fluid-solid heat transfer), whose definition requires the need of having a characteristic dimension of the system. The dimensional analysis of the porosity allows to characterize a specific foam structure, which in turns makes feasible the comparison between different types of foams. The parameters characterizing the foam structure are listed below:

- the cell size, equal to the average diameter of the empty space defined by the cellular structure;

- the pore size, equal to the average width of the windows that connect two contiguous cells;

- the size of the structure, which is a measure of the average diameter of the reticle;

- the porosity.

\subsection{Dimensional characterization by means Mathematical Morphology}

The methodology proposed in this work, which is based on the imaging analysis concept, comes from the intention to overcome the necessity of having available a file in the stl format, resulting by a micro-tomography. With this new approach a single two-dimensional or three-dimensional image of the structure under consideration is needed only. The use of a simple image is possible applying both the techniques of image processing and image analysis on the domain being studied. This new approach was applied for the first time in the present work for characterizing the geometry of an actual physical element, such as a metallic foam. The mathematical morphology derives from the theory of sets and was developed with the intent to process the image; it is adaptable to a wide range of industrial applications $[15,16]$. The purpose of this field is to obtain information by adopting procedures that make simple the reading of the images. The image to be analysed can be binary, gray-scale or both: the basic concepts can be applied to different classes of images. Processing is made via the definition of a structuring element chosen by the user: it represents a basic geometric profile, whose shape depends on the analysis to be performed. In essence, the resulting image is a non-linear transformation, produced by the interaction between the original image and the chosen structuring element.

In [15] all the elementary operations required by the mathematical morphology theory are listed. This theory is based on mathematical filters applied to discrete samples, i.e. images for example: they are non-linear translation-invariant images or signal transformations. Mainly there are two kinds of images: gray-tone and binary. In the following description we will refer to the concept of set to indicate the binary image, while the concept of function is related to the gray-tone image. More details can be found in [15]. Binary discrete images are represented by 2-D sets in the discrete plane $Z^{2}$, where $Z$ is the set of integer numbers and $R$ is that of the real ones. The following elementary operations used in the present study are defined: 
- Translation. Given a set $A \subseteq Z^{2}$ of points $a \in A$ and a point $p \in Z^{2}$ it is defined translation the set $A_{p}$ of points $a+p$ :

$$
A_{p}=\{p+a: a \in A\}
$$

- Symmetric. Given a set $A \subseteq Z^{2}$ of points $a \in A$ it is defined symmetry of $A$ the set $A^{s}$ such that:

$$
A^{s}=\{-a: a \in A\}
$$

- Erosion. Given a set $A \subseteq Z^{2}$ of points $a \in A$ and a structuring element $X \subseteq R^{2}$, the erosion result is the set of points $p \in Z^{2}$ such that:

$$
X \ominus A^{s}=\left\{p \in Z^{2}: A_{p} \subseteq X\right\}
$$

- Dilation. Given a set $A \subseteq Z^{2}$ of points $a \in A$ and a structuring element $X \subseteq R^{2}$, the dilation result is the set of points $p \in Z^{2}$ such that:

$$
X \oplus A^{s}=\left\{p \in Z^{2}: A_{p} \cap X \neq 0\right\}
$$

In details, combining erosion and dilation, spheres having different diameters are used for the evaluation of the pore size of the foam sample, returning a distribution of diameters related to the frequency with which they appear. The combination of these basic operations can generate more complex computations. For instance, the opening operation is the result of the application in series of the erosion operation, followed by the dilation one. Considering a structuring element $X$ on a set $A$, the opening operation is given by:

$$
X_{A}=\left(X \ominus A^{s}\right) \oplus A
$$

Although the operations just described are related to the 2-D images, the same considerations can be extended to threedimensional domains. An image composed by "three-dimensional pixels" may be subjected to the same procedures: discretization of the fluid domain defined by the solid geometry of the foam and application of the filters. Applying the above described methodology in three-dimensions it is possible to determine the cells and pores size of the foam sample. The whole process includes:

- Discretization of the foam domain into appropriate size cubes: small cubes will lead to a more precise geometry definition but will increase the computational cost.

- Definition of the structuring elements $S_{\text {struct }}$, which will be spheres of different diameter and will be also discretized into cubes of the same size used for the fluid domain.

- Application of erosion and dilation operations, using increasingly smaller structuring elements and eliminating, at each iteration, the volume of the domain resulting from the filtering process performed with the element $S_{\text {struct }}$ of a given size.

- Counting how many elements $S_{\text {struct }}$ of a given size are resulted from the whole process.

Through a Boolean operation performed using a CAD code, the fluid domain was extracted by the original foam sample. It was necessary to perform a discretization of the volume fluid, which represents the three-dimensional image to be studied (Figure 2). The discretization process was done by the mesh generator of the OpenFOAM ${ }^{\circledR}$ code (snappyHexMesh). As structuring element a series of spheres of different sizes has been chosen. This allows to verify the size of the cavities of the foam. Figure 3 shows an example of a spherical structuring element. All the spheres have been discretized with the same method: each spherical sub-sample was characterized by cubes of $0.2 \mathrm{~mm}$ side. The critical point of this process is the size of the cubic elements since the measuring accuracy and the computational cost depend on it. A good compromise has led to the determination of the measurement sensitivity in $0.2 \mathrm{~mm}$, which represents about the $8 \%$ of the mean dimension given by the supplier (10ppi equal to $2.54 \mathrm{~mm}$ ). The resulting porosity $\epsilon$ of the discretized fluid domain was:

$$
\epsilon=\frac{V_{f}}{V}=82.5 \%
$$

which well approximates the porosity of the actual solid sample in Table 1.

The tested diameters were varied in the range between $0.7 \mathrm{~mm}$ and $5.5 \mathrm{~mm}$ in order to represent the whole extension of the distribution. The equations (1-4) have been implemented in an algorithm written in FORTRAN code without particular changes to the above cited expressions. This algorithm has the objective of determining the number of times that a sphere of a given diameter can be contained in the fluid domain.

Note that the symmetry operation is useless because of the symmetry of the spherical structuring element, while the translation operation has the function of moving the center of each spherical structuring element in every point of the fluid domain: for each translation operation it is necessary to check if the examined point is positive to the erosion operation, i.e. the equation (3) is satisfied. If the check is positive, then the dilation operation is performed. In this way it is possible to obtain: 


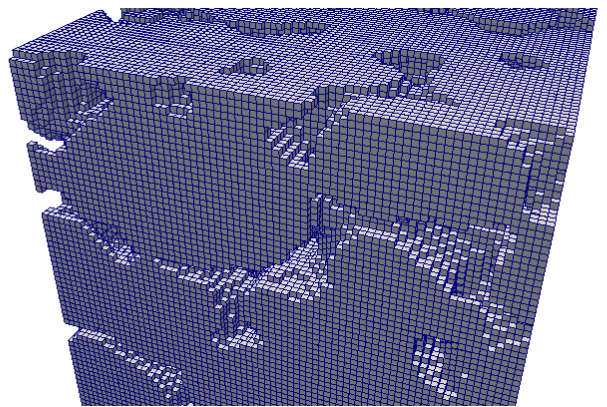

Figure 2: Discretization of the domain.

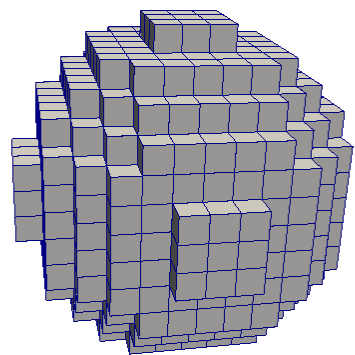

Figure 3: Discretization of the structuring element (sphere of $2.5 \mathrm{~mm}$ ).

- A list of the erosion points;

- A reconstruction of the geometry through the sample spheres used as a structuring element.

The algorithm examines the sphere of larger diameter, runs the above procedure and then rewrites the fluid domain coordinates discarding the dilation points eventually found. In this way, by repeating the analysis with the spheres of increasingly smaller size, there will be no overlapping points among spheres having different diameter. Note that the procedure excludes from erosion all the points outside the domain, since, due to the lack of knowledge about the foam geometry, it is not possible to verify if the structuring element is entirely contained. If $D_{m}$ is the diameter of the sphere having the largest diameter, the erosion points must be located at a distance smaller than $D_{m} / 2$ from each edge.

To reduce the computational cost of the procedure, it is useful to simplify the problem reducing it to a 2-D one: the fluid domain is reduced in parallel planes orthogonal to one of the main axes of the foam sample (Figure 4), while the spheres are in their turn transformed into discs of equal diameter. The 2-D analysis results are analysed hereinafter: note that the erosion dots indicate the location of the center of the spheres used for the reconstruction of the porosity of the foam. In Figures 5 and 6 the points of erosion determined by the 2-D analysis are shown: observing the larger diameters the placement of the centers appears to develop mainly along a direction (Figure 5), while the smaller diameters are spread much more chaotically (Figure 6). It can be assumed that the preferential direction, i.e. the one along which the largest pores are placed, could offer a lower resistance to fluid flow. This last result seems to suggest an anisotropy of the foam, which was verified and presented in the next paragraph.

In Figure 7 an example of erosion and dilation procedures is visible, searching for the diameter $d=2.5 \mathrm{~mm}$. The blue dots represents the fluid domain, the green dots are the points of the determined cells, i.e. having a diameter equal to $d=2.5 \mathrm{~mm}$. The white region of Figure 7 highlighted by the red line represents a previously filtered cell, having a diameter $d \geq 2.5 \mathrm{~mm}$. Figure 8 shows the dimensional distribution obtained by the 2-D analysis on $\mathrm{z}$-cutting planes.

Looking at the distribution of Figure 8 two probability functions are noticed: one with the mean value centred at about $1.5 \mathrm{~mm}$ and the other one at about $3.9 \mathrm{~mm}$. Moreover, the 2-D distribution shows to have the highest frequencies at the diameters $0.9 \mathrm{~mm}$ and $1.3 \mathrm{~mm}$. It should be noted that this data is compromised by three main aspects:

- Poor accuracy of measurement, because the discretization is carried out by cubic elements of $0.2 \mathrm{~mm}$. For measurements less than $1.3 \mathrm{~mm}$ they provide too low sensitivity. In fact, a single cube represents more than $15 \%$ of the measurement to be made. 


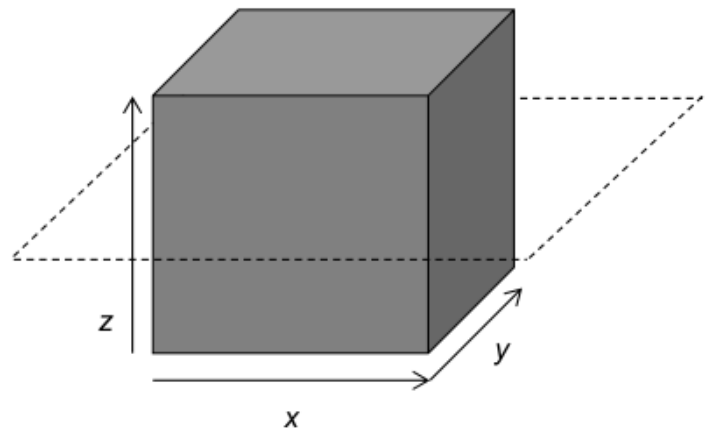

Figure 4: 2-D erosion by planes orthogonal to $\mathrm{Z}$ axis.

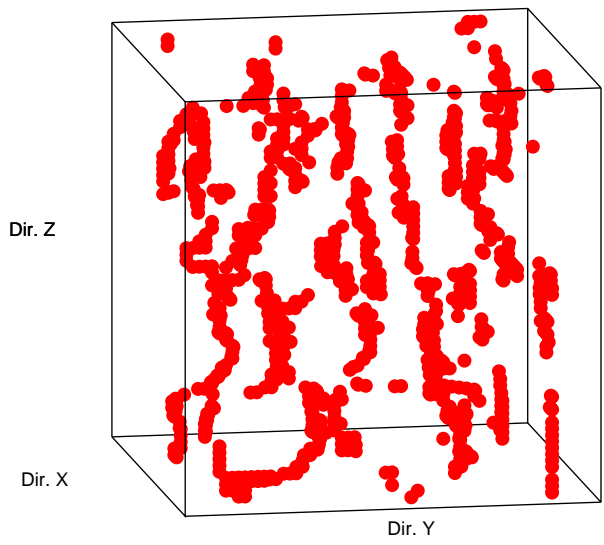

Figure 5: 2-D erosion points for larger diameters $(d \geq 2.3 \mathrm{~mm})$.

- Since the shape of each pore is not perfectly spherical (Figure 9), spaces being a residue of a porosity already counted could be detected as a new cavity.

- The used technique underestimates the maximum cell size $D_{m}$ in Figure 9, where the ideal porosity shape was supposed to be an ellipse in the 2-D domain and an ellipsoid in the 3-D domain.

For these reasons the frequencies found for diameters smaller than $1.5 \mathrm{~mm}$ are to be considered unreliable and therefore discarded. It is not simple to prevent or reduce this measurement error; as a matter of fact, the increase of the measurement sensitivity would lead to a higher accuracy but without eliminating the "false" porosity. In any case, there would be an increase in the computational cost. The problem lies in the irregular shape of the cavity for which a spherical approximation is acceptable but not precise. The usage of different volumetric shapes, for example ellipsoidal, it is possible but would complicate the problem by introducing new control parameters, such as the relations between the ellipsoid axes and the orientation of these axes with respect to the foam sample, which are variables obviously not known in advance.

For most accurate results the 3-D analysis is required. The erosion points defined by two-dimensional analysis were therefore processed by repeating the same procedure but in three dimensions: in this way only the points that have been shown to have the size necessary to represent the pore size are processed. In fact the two-dimensional condition is necessary but not sufficient to show that a sphere of a certain diameter can be contained in a certain pore. Moreover, in this way the computational cost is greatly reduced as the number of points to be processed decreases by at least an order of magnitude. Figure 10 shows the result of the 3-D implemented algorithm: the larger the sphere diameter, the lower the frequency of appearance in the discretized fluid domain. The distribution trend in the 3-D domain is similar to the one obtained by the 2-D analysis (Figure 11), at least up to the diameter of $2.5 \mathrm{~mm}$, which is close to the mean value of 10 ppi $(2.54 \mathrm{~mm})$ specified by the supplier as density of pores. Looking at the direct comparison of Figure 11, two issues are observed: 


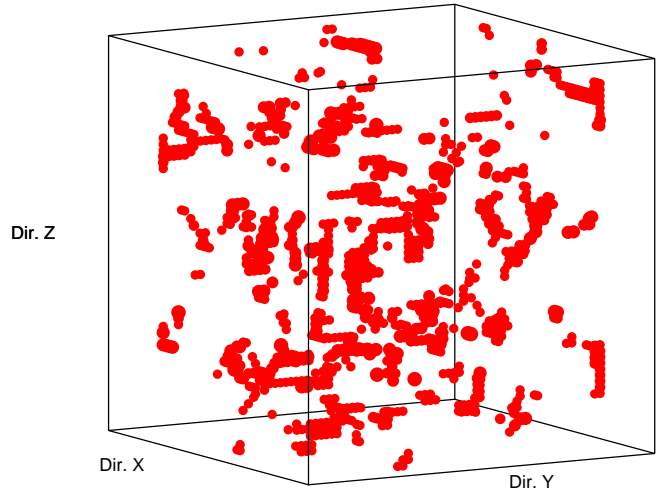

Figure 6: 2-D erosion points for smaller diameters $(d \leq 2.1 \mathrm{~mm})$.

- Calling $D_{2}$ the diameter computed by the 2-D analysis and $D_{3}$ the one measured by the 3-D analysis, it is possible to determine a correlation between 3-D and 2-D analysis. In fact, for each significant frequency detected in the 3-D analysis, a corresponding 2-D frequency exists, such that the ratio between the respective diameters of the porosity is maintained approximately constant and equal to 1.4. It is possible to state that the diameters distribution between 2-D and 3-D analysis is "scaled" by a factor which is in the range between approximately 1.3 and 1.5. This factor indicates that the shape of the pores is not spherical but rather ellipsoidal, with a ratio between the major and minor axes approximately constant. In order to explain these results, the generic porosity is idealized with an ellipse (for the pores) or with an ellipsoid (for the cells), as in Figure 9. The cells can be considered as ellipsoids with two semi-axes of about the same size and equal to $D_{2} / 2$, while in the $\mathrm{Z}$ direction there is an axis equal to $D_{3} / 2$. Ellipses are considered for the pores, having a ratio between the major and minor semi-axes equal to $D_{2} / D_{3}$. It is also to note that the used technique underestimates the maximum size of the cell $D_{m}$.

- The peak observed in the 2-D analysis due to the previously described distortion is significantly reduced in Figure 10, proving that the frequencies at relatively small diameters are not completely reliable.

In Figure 10 it is interesting to note the high frequency obtained for the diameter value $2.7 \mathrm{~mm}$, which is close to the mean size of 10 ppi representing the density of pores. Using a probability distribution function and combining the results obtained in 2-D and 3-D, it is possible to:

- Derive approximately the mean size of the cavities of the tested foam sample;

- Choose the most appropriate characteristic size to be used in the non-dimensional simulated physical quantities.

To conclude, are to be highlighted the following items:

- The 2-D distribution has been required to reduce the computational cost. It is definitively incomplete since nor the geometry of the cavity or the orientation of the sample compared to the reference system were known a priori: this could lead to misleading results. In the analysed case, being the pores ellipsoidal, one of the dimensions is substantially smaller than the others. This explains how the 3-D analysis detects both lower porosity values and sizes than the 2-D analysis. Then the 3-D analysis provides the most realistic size distribution of the porosity.

- Moreover, the comparison between 2-D and 3-D performance should take into account two factors:

- The geometry of the porosity is not spherical, in particular for what concerns the cells which, as already pointed out, are ellipsoidal. The 2-D analysis has detected the size of the main axes, as shown in Figure 9. The size of the pores, intended as the openings connecting contiguous cells, is less than the size of the cells, and then "populates" the smallest diameters of the distribution.

- The measurements of the smallest diameters are less accurate due to the adopted resolution, that is the size of the cubes used for the domain discretization.

- For diameters greater than $2.5 \mathrm{~mm}$, the 2-D and 3-D trends show to be in a certain ratio (an average value of about 1.4), which should represent the ratio between the major and the minor axes of the cells 


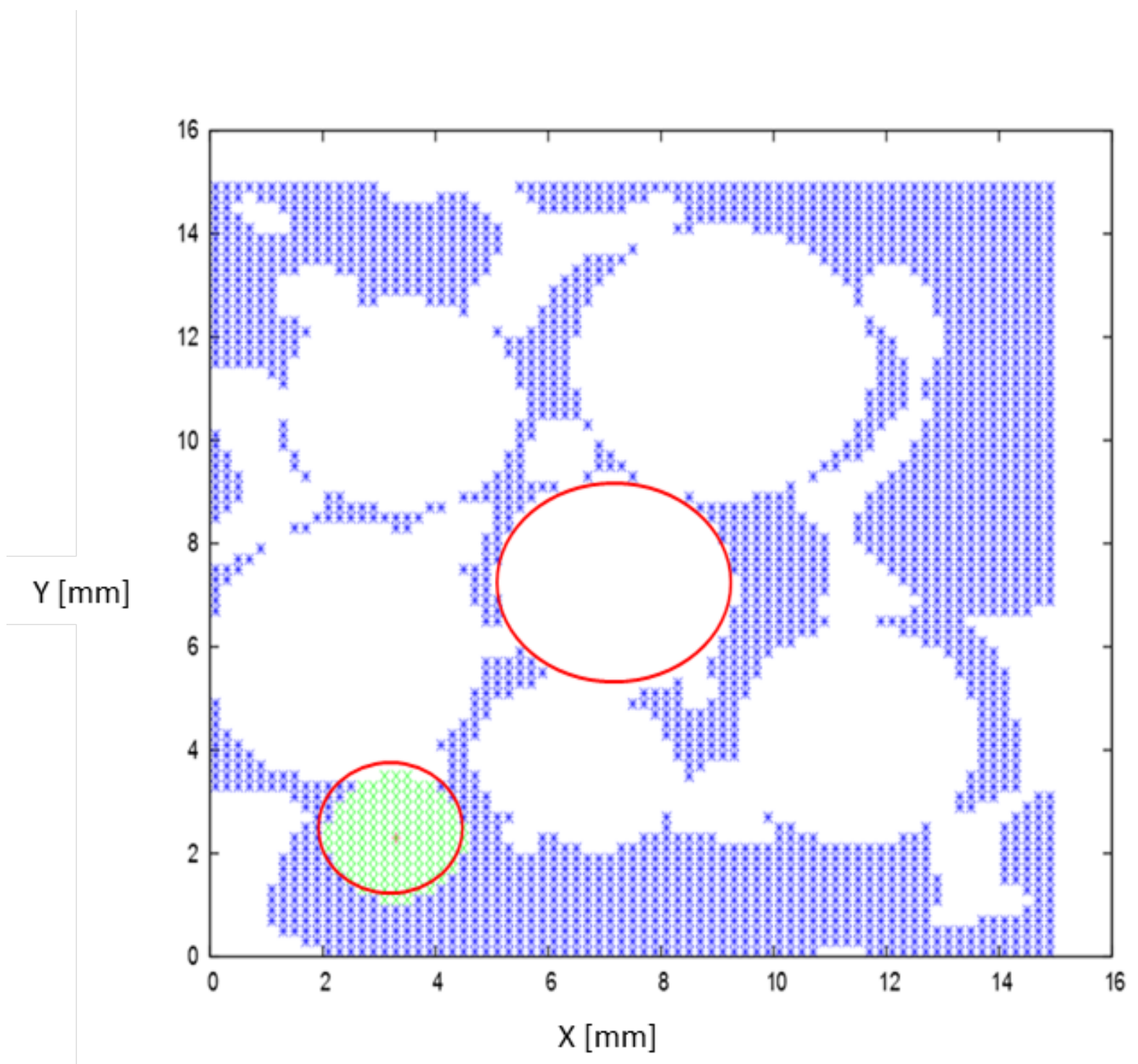

Figure 7: 2-D erosion and dilation example $(d=2.5 \mathrm{~mm})$.

(Figure 9). For diameters smaller than $2.5 \mathrm{~mm}$, this ratio becomes gradually less appreciable because the size of the pores, characterized by a more circular shape, is now detected.

- It is well known that the first peak of the distribution identifies the pore size, the second peak instead the cell size. By the results (Figure 10) an acceptable mean cell size can be assumed to be $2.54 \mathrm{~mm}$ (10 ppi).

\section{Fluid-dynamic characterization of metallic foams}

Once defined the geometrical characteristic of the foam sample (pore and cell sizes), for a given input velocity it is possible to determine more correctly the flow regime. The CFD simulations were performed by the OpenFOAM ${ }^{\circledR}$ code, with the following main set-up parameters/models:

- Laminar flow: the consistency of this choice was assessed and it was reported in the paragraph below. 


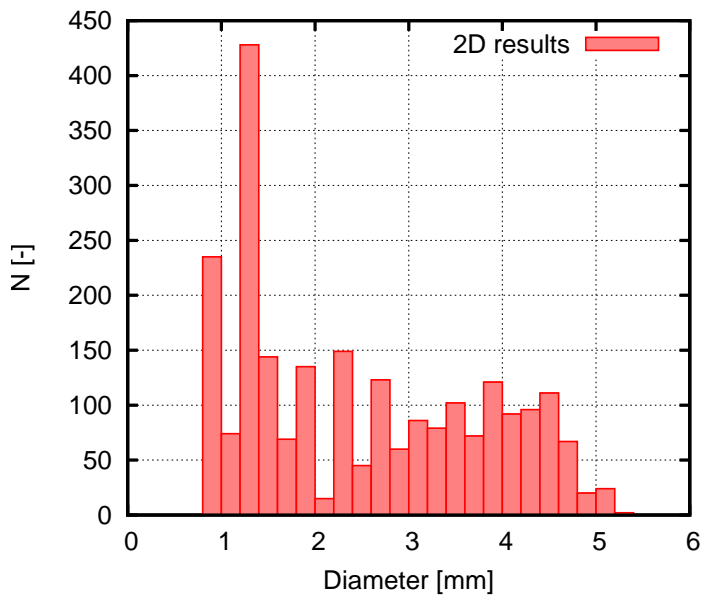

Figure 8: 2-D diameters distribution on multiple z-cutting planes.

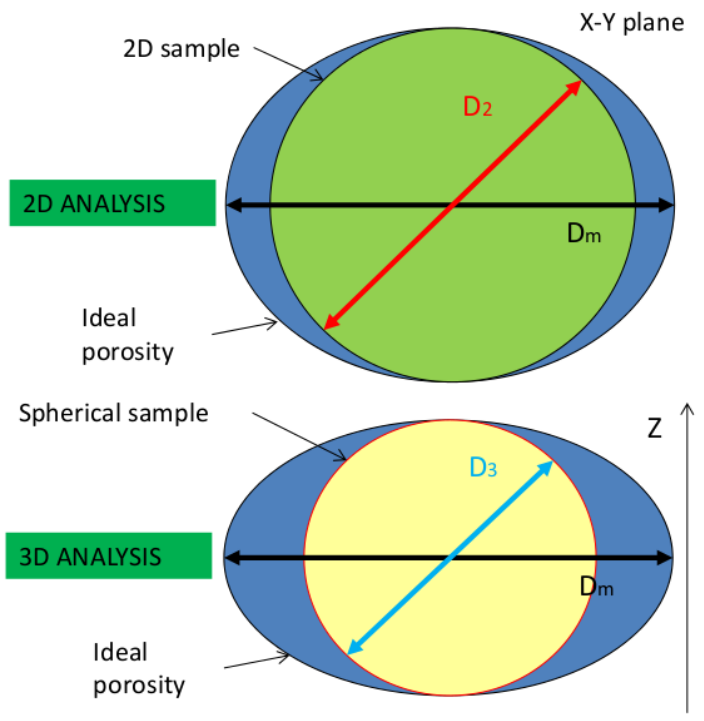

Figure 9: Ideal geometry of porosity.

- Ideal fluid. The working fluid in the present analysis was air, treated as an ideal gas. Both the hypotheses of compressible gas and viscous flow were satisfied: the basic equation of state was the perfect gas law. The main air physical parameters are listed in Table 2.

- Boundary conditions:

- INLET - The Darcy velocity was imposed, computing its value as the ratio between the volumetric flow rate and the frontal geometric section.

- OUTLET - Static pressure was imposed.

- On the foam surface the WALL condition was imposed.

- The symmetry conditions was imposed on the faces of the fluid sample.

The mesh main characteristics are reported in Table 3.

\subsection{Turbulence characterization of metallic open-cell foams}

Della Torre e al. $[8,10]$ focused their attention on the Kelvin cell structures. In the present paper the authors based their analysis on an actual foam sample, geometrically characterized as described in the paragraph above. It was decided 


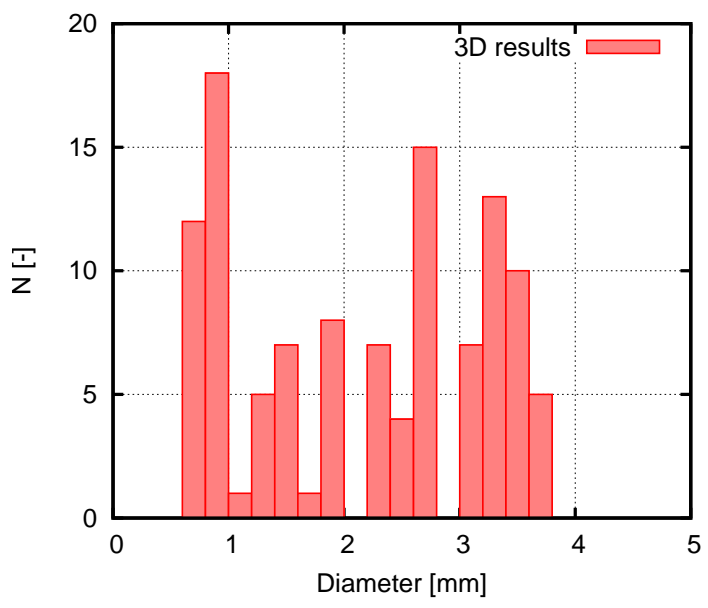

Figure 10: Erosion 3-D: diameters distribution.

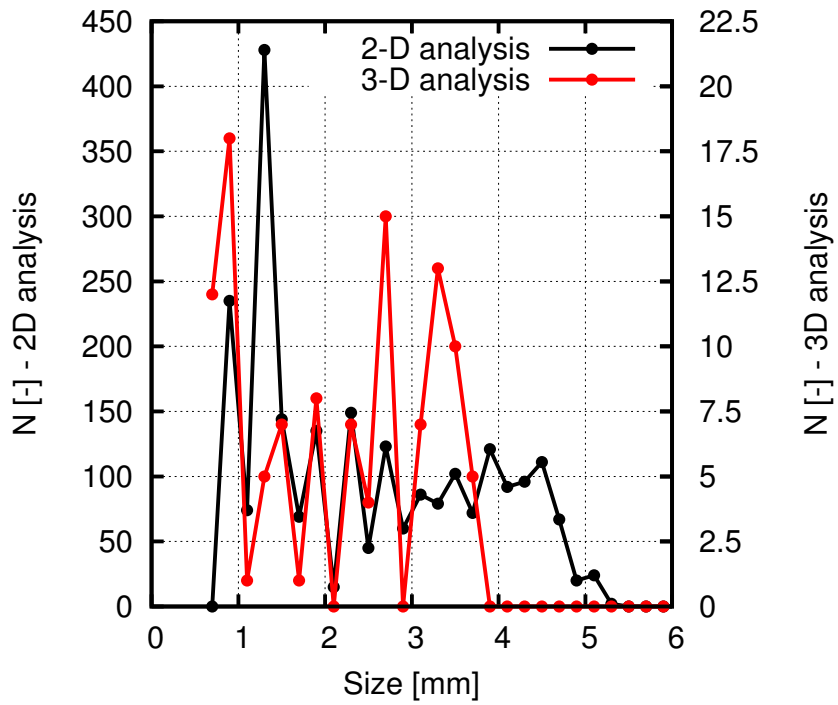

Figure 11: Diameters distribution for 2D and 3D erosion.

to adopt the $K-\omega$ SST model on the basis of the findings reported in [8]. Nevertheless four different models were implemented and tested on the actual foam sample to further validate the obtained results:

- Transition $K-\omega$ SST (4 equations), called Trans $K-w S S T$.

- $K-\epsilon R N G$ - Enhanced wall treatment - Called $K$ - epsilon RNG.

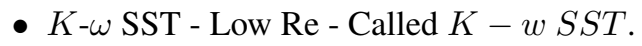

- Laminar.

The Reynolds number was pore-based $R e_{p}$, as usual. The Reynolds number values were examined up to 5000 : this wide range covers both classical production cars and cars designed for having high performances, including racing cars. For classical automotive applications the typical Darcy velocity of the exhaust gas flowing trough a catalytic device is less than $10 \mathrm{~m} / \mathrm{s}$, while for racing applications, due to the higher rotational engine speed, the Darcy velocity could reach values close to $50 \mathrm{~m} / \mathrm{s}$.

The CFD results are visible in Figure 12: there is an alignment of the pressure drop results. It is to note a slight difference between the results obtained with the models $K-\omega$ SST and $K-\epsilon$ : this is ascribable to a different approach of the wall treatment. Generally speaking, the pressure drop curve is independent by the choice of laminar or turbulence model: this result confirms the findings of Della Torre et al. $[8,10]$ based on the simulations of the Kelvin cells. 


\begin{tabular}{lc}
\hline$\rho\left[\mathrm{kg} / \mathrm{m}^{3}\right]$ & 1.1767 \\
$\mu[\mathrm{Pa} \cdot \mathrm{s}]$ & $1.8600 \cdot 10^{-5}$ \\
Pressure $p[\mathrm{~Pa}]$ & 101325 \\
Temperature $T[\mathrm{~K}]$ & 300
\end{tabular}

Table 2: Physical parameters of the working fluid.

\begin{tabular}{lc}
\hline Basic dimension $[\mathrm{mm}]$ & 0.4 \\
Minimum dimension $[\mathrm{mm}]$ & 0.1 \\
Prismatic layers at wall & 3 \\
Total thickness & \\
of wall layer $[\mathrm{mm}]$ & 0.06 \\
Total number of cells & $6.1 \cdot 10^{6}$ \\
March angle [ $\left.{ }^{\circ}\right]$ & 85 \\
\hline \multicolumn{2}{c}{ Table 3: Mesh main characteristics. }
\end{tabular}

Della Torre at. el. ([10]) showed that, depending on the pore-based Reynolds number, four different flow regimes can be distinguished in a generic porous medium: (a) Darcy, (b) Forchheimer, (c) post-Forchheimer, (d) fully turbulent regime. The most common model for describing the flow in the first two regimes is represented by the Darcy-Forchheimer relationship:

$$
\frac{\Delta p}{L}=\frac{\mu}{K} U_{D}+\rho C U_{D}^{2}
$$

The authors showed that for the four types of regimes Equation (7), suitably non dimensionalized, can be still considered valid if expressed as follows:

$$
\Pi_{1}=A+B\left(R e_{p}\right)=\mathcal{F}_{1}\left(R e_{p}\right),
$$

where $A$ and $B$ are function of permeability $K$ and form coefficient $C$. The non-dimensional $\Pi_{1}$ is a tensor representing the flow resistance in the three main directions.

For post-Forchheimer and fully turbulent regimes the same equation can be considered valid if corrected coefficient $\mathrm{A}$ and B are used: $A_{\text {turb }}+B_{\text {turb }}$.

$$
\mathcal{F}_{1, \text { turb }}(R e)=A_{\text {turb }}+B_{\text {turb }}\left(R e_{p}\right) .
$$

On the basis of these findings, the fitting curve expression was derived by Equation (7) and rewritten as follows:

$$
\frac{\Delta p}{L}=k_{v} U_{D}+k_{i} U_{D}^{2},
$$

where the viscous coefficient $k_{v}$ value is:

$$
k_{v}=148.53 \frac{\mathrm{Pas}}{\mathrm{m}^{2}},
$$

and the inertial coefficient $k_{i}$ value is:

$$
k_{i}=322.4 \frac{\mathrm{Pas}^{2}}{\mathrm{~m}^{3}} .
$$

Figure 13 shows the comparison of the simulation results obtained in the present work with the experimental measurements reported in [17]. The available experimental result refers to a $\mathrm{SiC}$ foam having similar porosity (85\%) but higher pore density (cell diameter equal to $d_{c}=1.35 \mathrm{~mm}$ ): in order to make it possible the comparison, results are plotted in terms of non-dimensional pressure drop $\Pi_{1}$. The comparison shows a reasonable agreement, confirming the validity of the overall simulation procedure.

In Figure 14 the fitting curve for the pressure drop is visible: also in the fully turbulent regime the fitting curves determines the trend of the pressure drop with a good accuracy. These data come from CFD simulation of the actual foam sample. A deep insight of these results is carried out at this point for a better understanding. As stated before, the pressure drop over the whole range of variation of the pore-based Reynolds number is the same, independently from the choice of the laminar rather than the turbulent model. The Reynolds number chosen for the analysis of this result was 932 , computed by scaling the Reynolds number by the effective face surface (fully turbulent flow regime). In particular CFD results by laminar flow regime and turbulent flow regime ( $K-w S S T$ turbulence model) were compared. A plane orthogonal to the Y-direction of Figure 4 was chosen, at $y=7.5 \mathrm{~mm}$ from the origin.

In Figure 15 the pressure flow field for laminar flow is reported, while in Figure 16 it is visible the corresponding image for turbulent flow. In Figures 17 and 18 the results of the CFD calculations in terms of velocity magnitude were reported respectively for laminar and turbulent flow. It is possible to note that: 


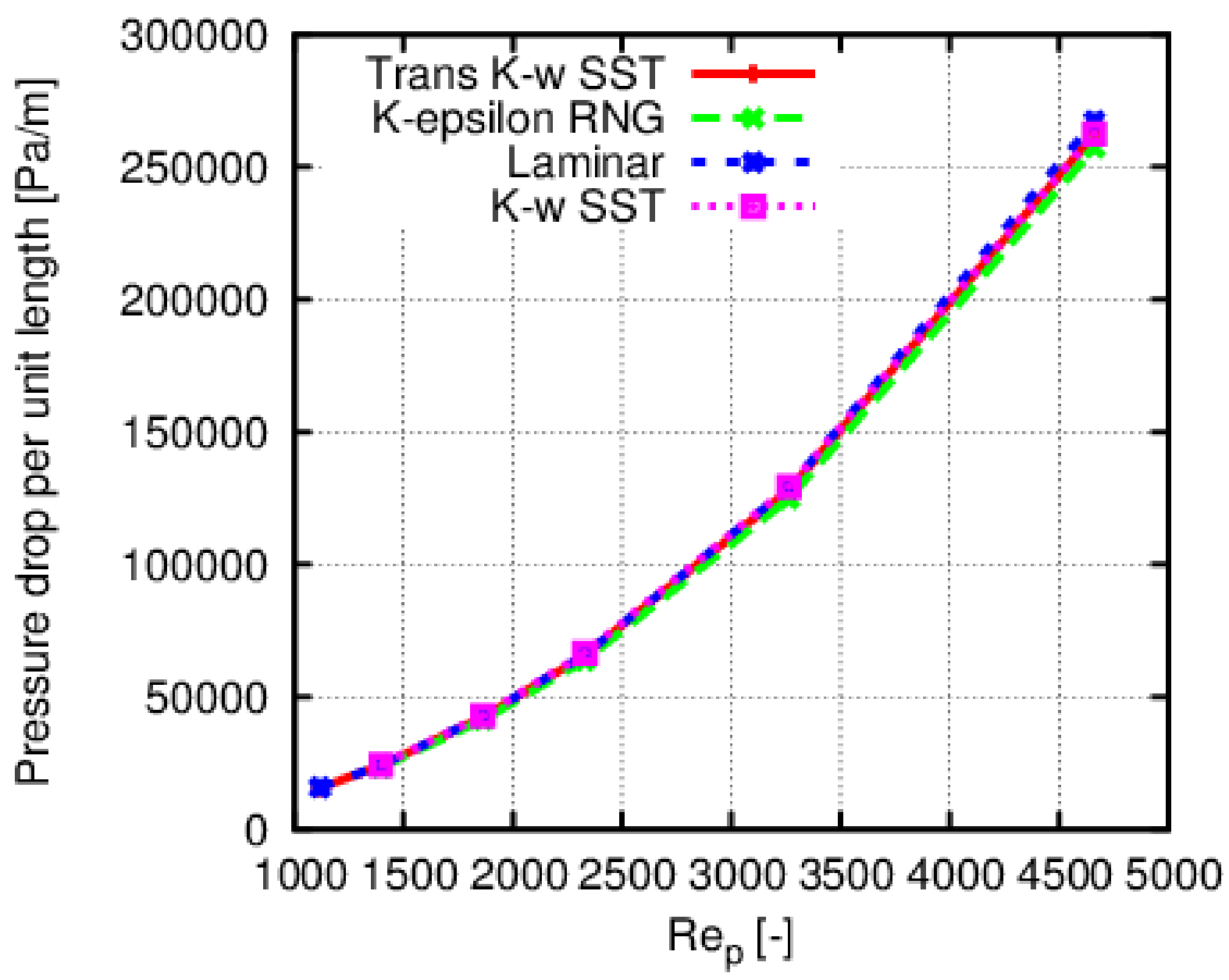

Figure 12: Pressure drop vs $R e_{p}$ for different turbulent models and laminar condition.

- The flow inside the foam sample is not necessarily laminar.

- The main contribution to the overall pressure gradient is linked to the inertial effect due to the tortuous flow path, i.e. the continuous variation of the fluid inertia.

- The dissipative effect caused by viscous forces at high $R e_{p}$ plays a minor role since it represents less than $20 \%$ of the total. Its contribution is neither negligible nor marginal, but it is definitely not overwhelming.

- Turbulent viscous losses are less than $5 \%$ of the molecular losses.

- Overall, for the considered flow regimes, the effect of the turbulence on the pressure drop is negligible. Nevertheless there is turbulent development in the sample.

The comparison was summarized in Table 4, where the percentage variations of the pressure drop with respect to the laminar condition were reported: they were computed for the same mass flow rate.

\begin{tabular}{cc}
$R e_{p}$ & $\Delta p$ \\
\hline 466 & $1.3 \%$ \\
932 & $2.5 \%$
\end{tabular}

Table 4: Percentage differences compared to the results of the laminar case.

Following the same modelling approach but trying to overcome the simplicity of the Kelvin cells, the authors in [7] determined the trends of the coefficients $A$ and $B$ comparing two different actual samples of metallic foams, one in 


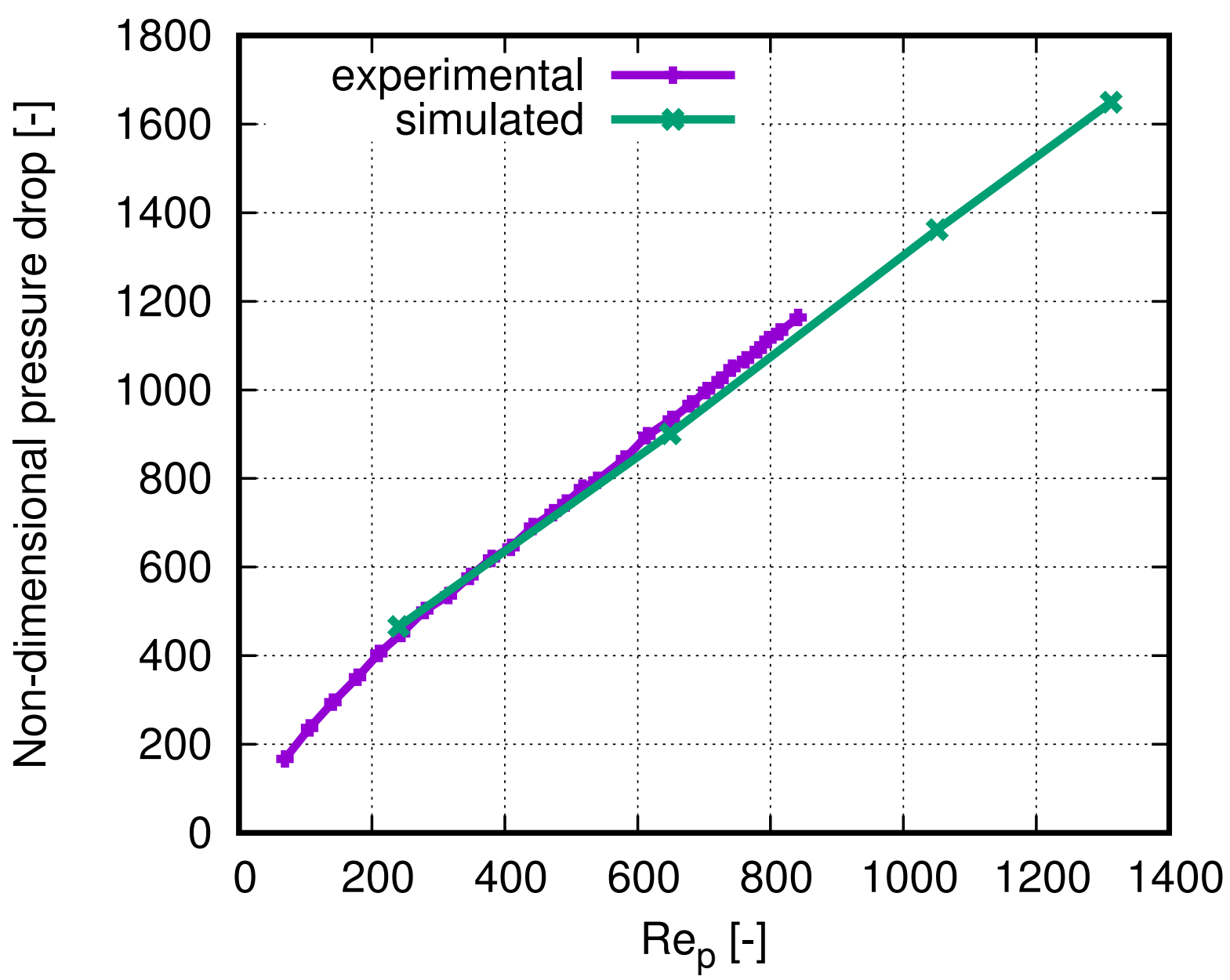

Figure 13: Comparison with experimental data: non-dimensional pressure drop $\Pi_{1}$.

silicon carbide, which is the same analysed in this paper (SiC-foam), and one in aluminium (Al-foam). The results found in [7] were reported in Figures 19 and 20 for clarity sake. In this way it was possible to characterize the contribution of the viscous and inertial effects on the pressure drop and relate the dependency of the pressure drop on the Reynolds number. Moreover, the dependency of the coefficients $A$ and $B$ on the porosity of the sample was investigated, in order to describe the relation between the geometry and the permeability of the foam. Comparing Figures 19 and 20 it is to note that the pressure drop is roughly the same between Al-foam and SiC-foam for the highest values of the porosity, while at the lowest values their difference becomes more significant. In particular these trends seems to suggest a less viscous resistance of the SiC-foam sample, which could be crucial for the pressure drop. For this reason in the present work the $\mathrm{SiC}$-foam sample was considered. Note that once the foam (metallic material) and its porosity have been defined, the coefficients $\mathrm{A}$ and $\mathrm{B}$ are uniquely determined.

\subsection{Check of the anisotropy of the foam sample}

The pressure losses in the three flow directions have been evaluated on the SiC-foam sample. The Darcy-Forchheimer expression (7) was applied in the three main directions. For example for the X direction the equation (7) becomes:

$$
\frac{\Delta p}{x}=\frac{\mu}{K} u+\rho C u^{2}
$$

The results are shown in Figure 21. The foam sample shows to have a fluid-dynamic anisotropy, even if not relevant. In particular in the $\mathrm{Z}$ direction the lower pressure drop is present in accordance with the previous geometrical analysis (see Figure 5). The pressure losses are determined by intrinsic properties such as the porosity, the mean pore diameter 


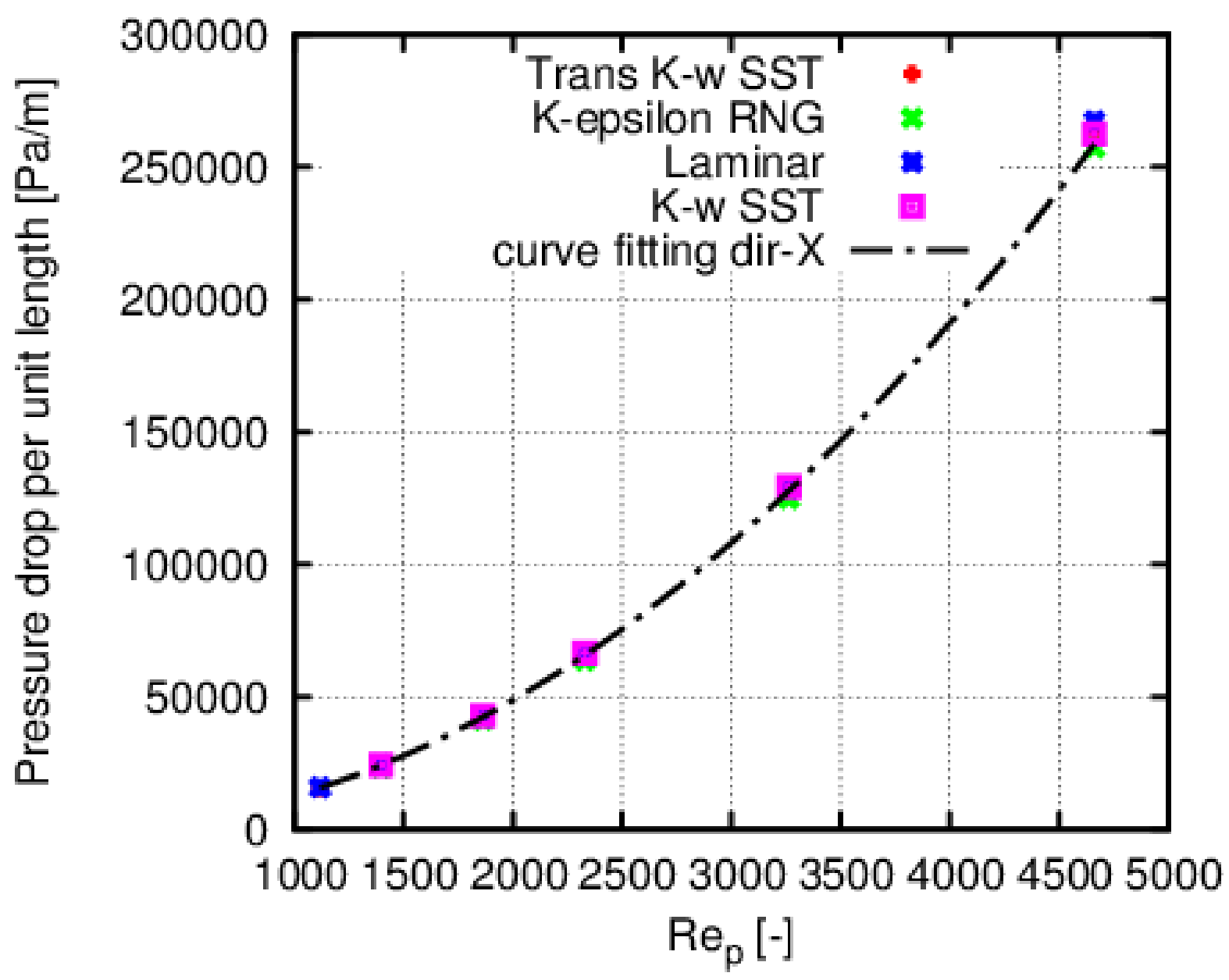

Figure 14: Pressure drop vs $R e_{p}$ - Fitting curve.

and the morphology: the pressure gradient was parametrized with respect to such properties on the given type of foam by means of coefficients $A$ and $B$.

\subsection{Effect of porosity and mean pore size on the foam fluid-dynamics}

For studying the effect of the porosity and the pore size the morphology of the foam was kept unchanged. Two operations were performed on the geometry:

1. Scaling: variation of the pore size at constant porosity (Figure 22);

2. Surface offset: variation of the porosity with pore size approximately constant (Figure 23);

The results were derived in terms of the Darcy friction factor $f$ variation, which is commonly used for describing the pressure drop in circular pipes, on pore-based Reynolds number $R e_{p}$ :

$$
f=\frac{2 d_{p} \Delta p}{\rho U_{D}{ }^{2} L}=2 \Pi_{2},
$$

where $L$ is the foam sample length. $\Pi_{2}$ is the equivalent expression of $\Pi_{1}$ in equation (8), using as repeating variables in the Buckingham theorem $\left(U_{D}, \rho, d_{p}\right)$, as reported in [8]. In Figures 24 and 25 the relationships between $f$ and $R e_{p}$ for the same morphology of the foam are visible. In Figure 24 different pore density values were considered, for a fixed porosity value equal to the one of the original sample (Table 1). In Figure 25 the porosity was varied keeping the 


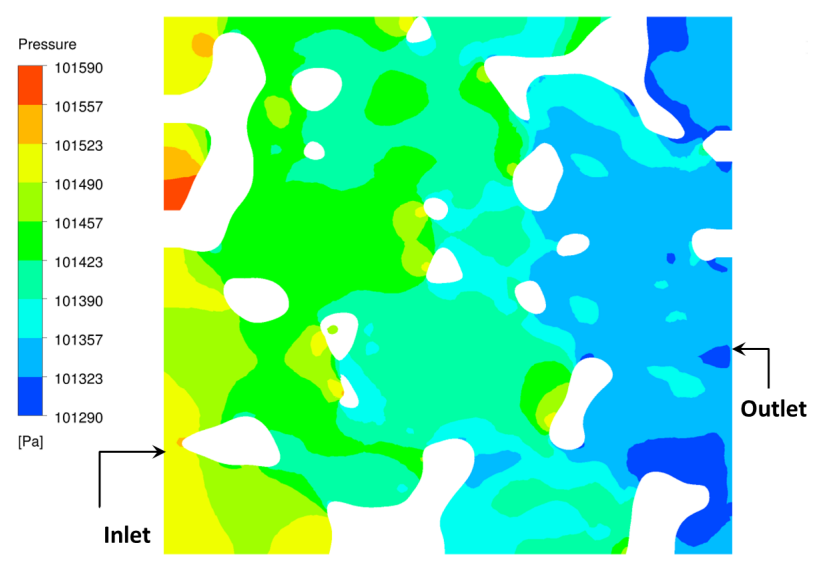

Figure 15: Pressure field - LAMINAR CASE.

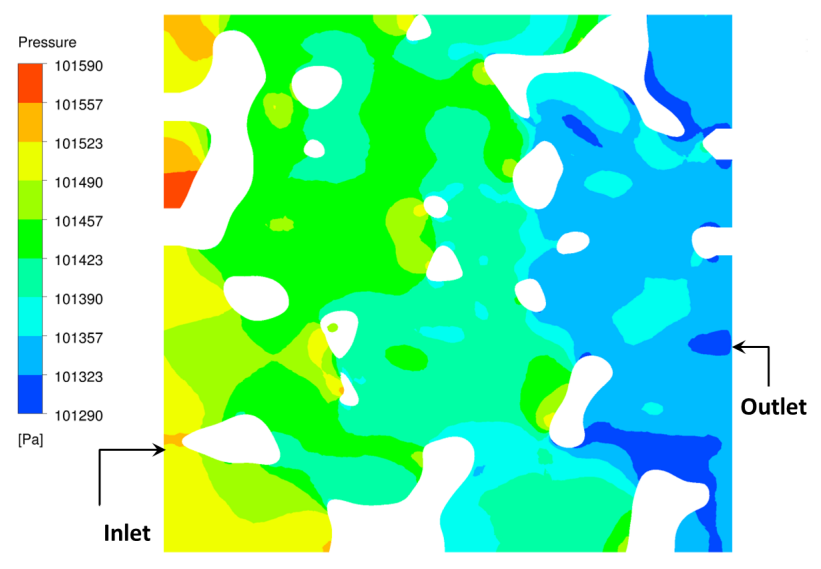

Figure 16: Pressure field - TURBULENT CASE.

pore density constant and equal to the value of Table 1 . Note that the friction factor $f$ is less influenced by the pore size than the porosity.

The same analysis was performed after the characterization of a traditional honeycomb strut, deriving the data from the open literature [18]. In Figure 26 the comparison between foam and honeycomb struts in terms of $f$ on $R e$ was reported: at this point it is necessary to point out that for the foam the term Re stands for pore-based Reynolds number, while for the honeycomb stands for the Reynolds number based on the hydraulic diameter. The porosity was $10 \mathrm{ppi}$ for $\mathrm{SiC}$-foam and 600 cpsi for the honeycomb strut.

Looking at Figure 26, it must be noted that, for reducing the flow resistances of the foam, it is possible to act on:

- Increasing the porosity $\epsilon$, which determines the shift of the curve downwards, toward the honeycomb curve;

- Decreasing the pore density which determines, for a given curve, the shift to higher $R e$ values, and then towards the flattening of the curve itself.

Last, it was verified the relationship between the specific surface and the porosity on one side, the specific surface and the pore density (ppi) on the other side. In Figure 27, where the dependence of the specific surface $S_{V}$ on the pore density is depicted, it is to note the linear trend: increasing the pore density, there is an increase of the specific surface. The limit to the pore density increase is due to the maximum allowed pressure drop for automotive applications. Instead, the trend of the relationship between the specific surface and the porosity $\epsilon$ is exponential, as shown in Figure 28:

$$
S_{V}=\alpha_{1}\left(1-\exp \left(\alpha_{2} \epsilon\right)\right),
$$




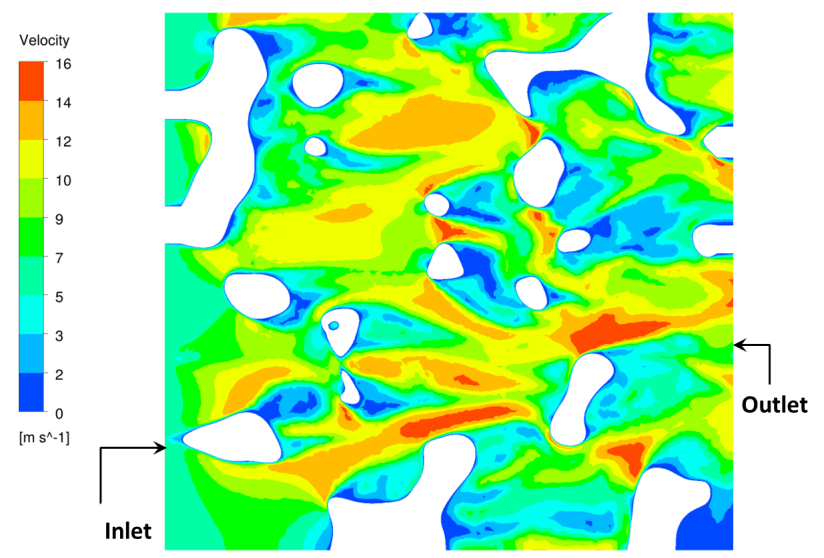

Figure 17: Velocity field - LAMINAR CASE.

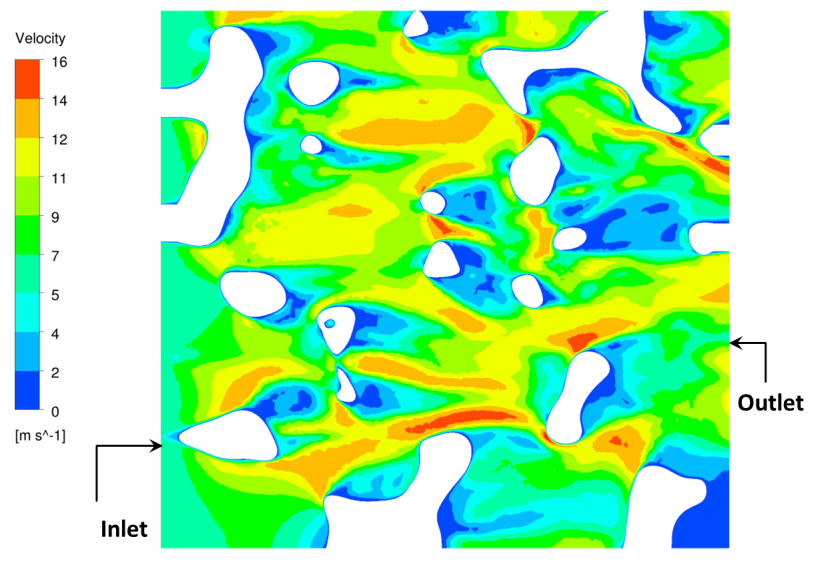

Figure 18: Velocity field - TURBULENT CASE.

the more the porosity of the foam, the less its specific surface. It is to remind that the importance of a high value of the specific surface is related to the possibility of reducing the loading of noble metals in the catalytic devices for automotive applications. Moreover, the strut morphology is crucial not only for the computation of the pressure gradients, but also for the extension of the specific surface of each foam [19]. There is a relation linking the coefficient $A$ to the specific surface $S_{V}[7,8]$ :

$$
-\sqrt{1 / A} \propto S_{V}
$$

From Equation (16) it is possible to state that the decrease of the specific surface leads to an increase of the permeability, correlated to the coefficient $A$. For the same flow regime and for the same porosity $\epsilon$, different values of $A$ involve different values of the specific surface mainly due to the different morphology of the foams.

\section{Conclusions}

The geometrical characterization of the foam sample, i.e. the statistical evaluation of the size of the cells and the pores was necessary to determine more correctly the flow regime for a given inlet flow rate. A procedure allowing the statistical computation of the number of cells and pores of a given size has been implemented in order to obtain a diameters distribution. Since a micro-tomography of the sample is not always available, the first part of the work aimed to illustrate a new methodology based on the imaging analysis. It allows to geometrically characterize the foam using a simple 2-D or 3-D image, in whatever format it is. The analysed foam sample was an open-cell foam made by 


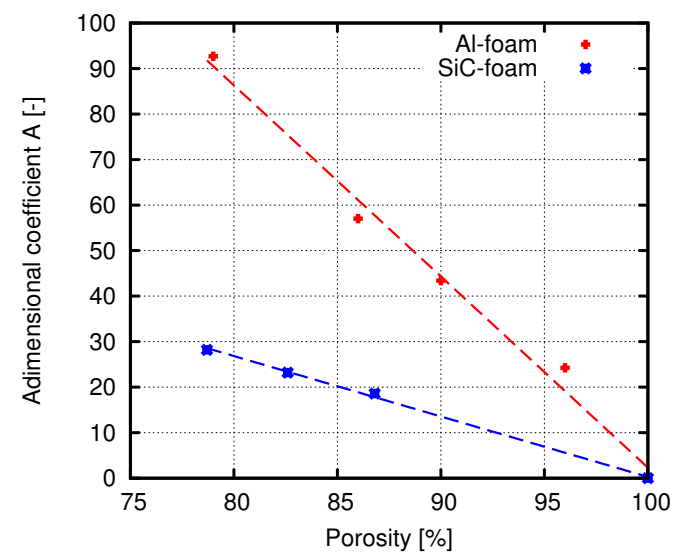

Figure 19: Adimensional coefficient $(A)$ for $\mathrm{Al}$ and $\mathrm{SiC}$ foams - viscous contribution [7].

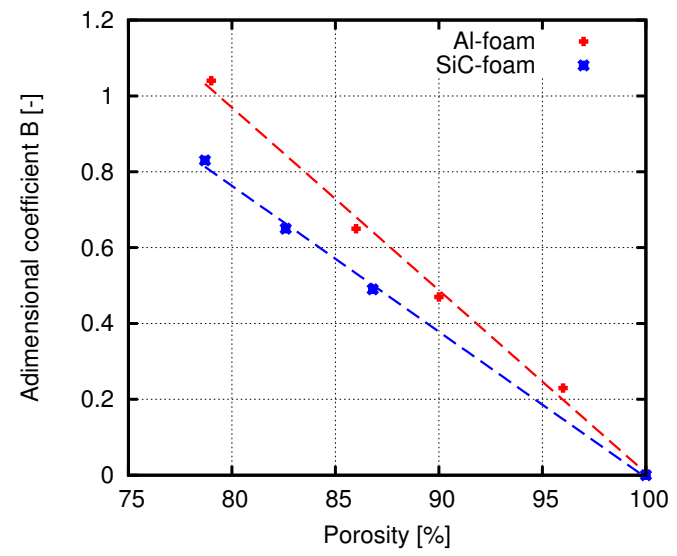

Figure 20: Adimensional coefficient $(B)$ for $\mathrm{Al}$ and $\mathrm{SiC}$ foams - inertial contribution [7].

silicon carbide $\mathrm{SiC}$.

Being the pressure losses determined by intrinsic properties such as porosity and mean pore diameter, the effect of variation of these parameters was investigated keeping constant the morphology. The actual original foam sample was subjected to two operations: scaling and surface offset. The results were summarized in terms of Darcy friction factor showing that it is less influenced by the pore size than the porosity. The analysis of the porosity shows a wide size distribution of the passage sections available to the fluid flow, therefore the detection of a characteristic dimension is not simple. The first peak of the distribution identifies the pore size, the second peak instead the cell size. By the results an acceptable size can be assumed $2.54 \mathrm{~mm}(10 \mathrm{ppi})$. The arrangement of the largest porosities seems to suggest a preferential direction for the flow. Moreover, comparing the distributions obtained from 2-D and 3-D analysis a similar pattern of the distributions was found but with a ratio ranging between about 1.3 and 1.5 , which suggests an ellipsoidal shape of the cells. Therefore as previously suggested by the results of the geometric characterization and then confirmed by this last analysis, the flow resistances of a foam can be reduced acting on:

- Porosity increase;

- Decrease of the pore density which determines, for a given curve, the shift to higher $\operatorname{Re}_{p}$ values, and then towards the flattening of the curve itself.

The geometric characterization was then applied to determine the flow regime: the average pore size $d_{p}$ was assumed to identify the characteristic dimension of the geometry. The Reynolds number was set to the pore-based Reynolds number $R e_{p}$ and the resulting flow regimes were fixed.

Regarding the turbulence analysis of the foam sample, the main results were:

- The main contribution to the overall pressure gradient is due the inertial effect caused by the tortuous flow path, i.e. the continuous variation of the fluid inertia. 


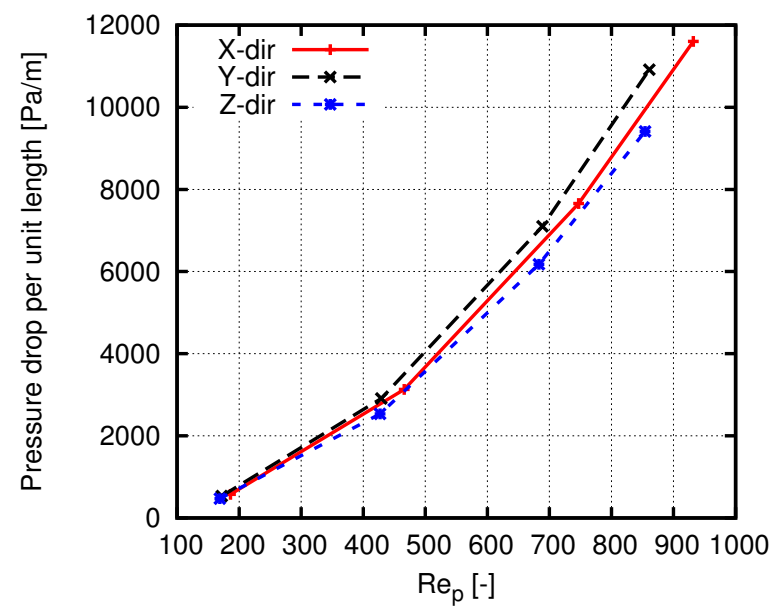

Figure 21: Pressure drop per unit length vs $R e_{p}$ in the $\mathrm{x}-\mathrm{y}-\mathrm{z}$ directions.

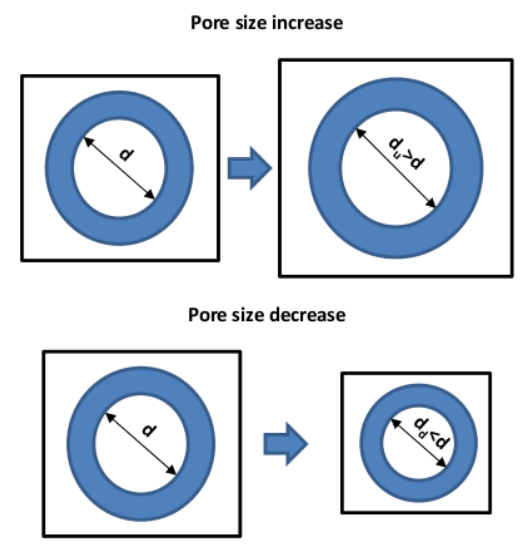

Figure 22: Pore size variation - Scaling operation.

- For the considered flow regimes the effect of the turbulence, though presents, on the pressure drop is negligible.

This is due to the fact that, at the flow regimes in which turbulence appears, the flow inside the porous matrix is dominated by inertial effects, while the viscous ones play a minor role. This means that the pressure drop is mainly determined by the inertial contribution and is not significantly affected by the increase of the viscous stresses promoted by the turbulence.

\section{Acknowledgements}

The authors gratefully acknowledge financial support from the Italian Ministry of Education, University and Research, Rome (MIUR, progetti di Ricerca Scientifica di Rilevante Interesse Nazionale, prot. 2010XFT2BB), within the project IFOAMS: Intensification of Catalyitic Processes for Clean Energy, Low-Emission Transport and Sustainable Chemistry using Open-Cell Foams as Novel Advanced Structured Materials.

\section{Contact Information}

stefania.falfari@unibo.it gianluca.montenegro@polimi.it 

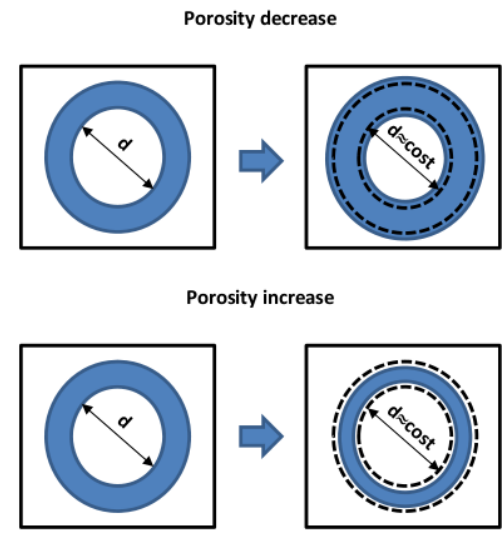

Figure 23: Porosity variation - Surface offset operation.

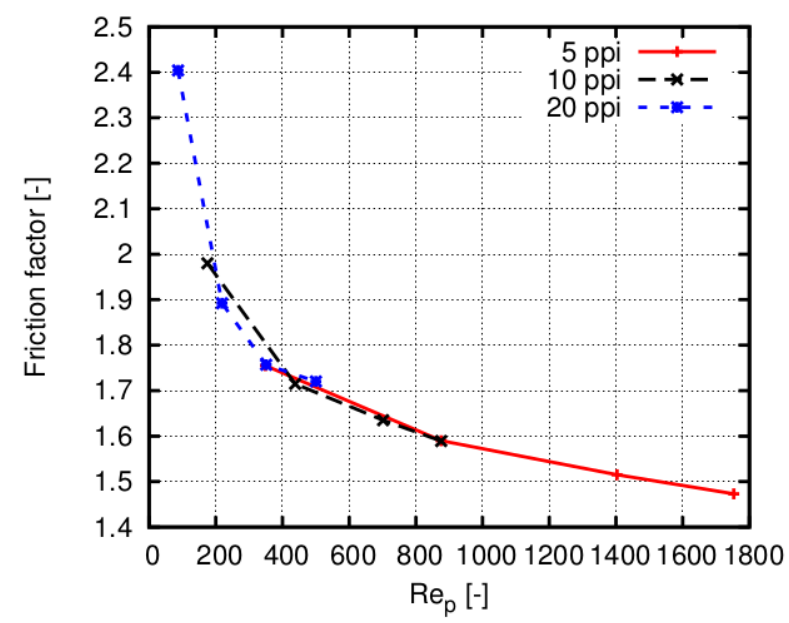

Figure 24: Friction factor versus Reynolds number for different pore size values.

\section{References}

[1] C. Bach and P. Dimopoulos Eggenschwiler. Ceramic foam catalyst substrates for diesel oxidation catalysts: Pollutant conversion and operational issues. SAE Technical Papers, 2011.

[2] A. Della Torre, F. Lucci, G. Montenegro, A. Onorati, P. Dimopoulos Eggenschwiler, E. Tronconi, and G. Groppi. Cfd modeling of catalytic reactions in open-cell foam substrates. Computers and Chemical Engineering, 92:5563, 2016.

[3] E. Bianchi, T. Heidig, C.G. Visconti, G. Groppi, H. Freund, and E. Tronconi. An appraisal of the heat transfer properties of metallic open-cell foams for strongly exo-/endo-thermic catalytic processes in tubular reactors. Chemical Engineering Journal, 198-199:512-528, 2012.

[4] D. Seguin, A. Montillet, and J. Comiti. Experimental characterisation of flow regimes in various porous media-i: Limit of laminar flow regime. Chemical Engineering Science, 53(21):3751-3761, 1998.

[5] D. Seguin, A. Montillet, J. Comiti, and F. Huet. Experimental characterization of flow regimes in various porous media-ii: Transition to turbulent regime. Chemical Engineering Science, 53(22):3897-3909, 1998.

[6] M.J.S. de Lemos. Turbulence in Porous Media. 2006.

[7] S. Falfari, G. Micci, G.M. Bianchi, F. Brusiani, G. Montenegro, A. Della Torre, and A. Onorati. Design of catalytic devices by means of genetic algorithm: Comparison between open-cell foam and honeycomb type substrates. SAE Int. J. Engines, 9(1):500-500, 2016.

[8] A. Della Torre, G. Montenegro, G.R. Tabor, and M.L. Wears. CFD characterization of flow regimes inside open cell foam substrates. International Journal of Heat and Fluid Flow, 50:72-82, 2014. 


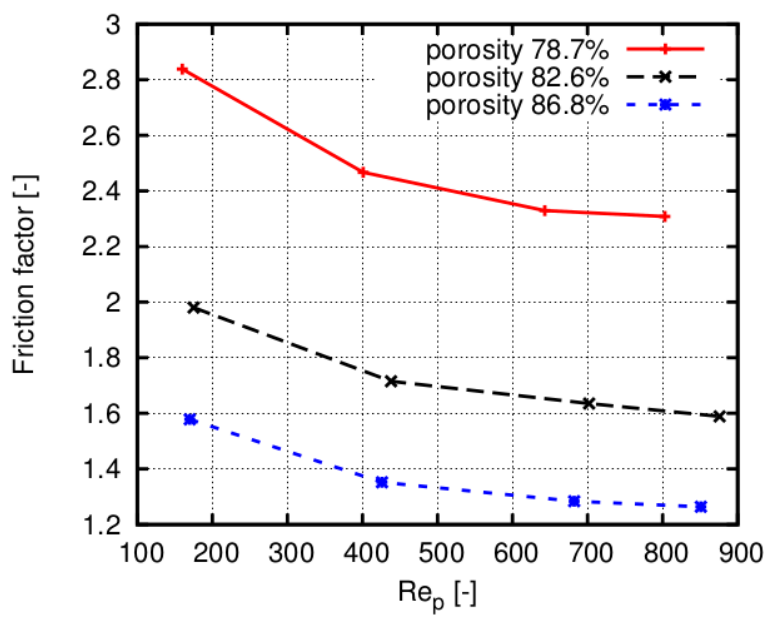

Figure 25: Friction factor versus Reynolds number for different porosities.

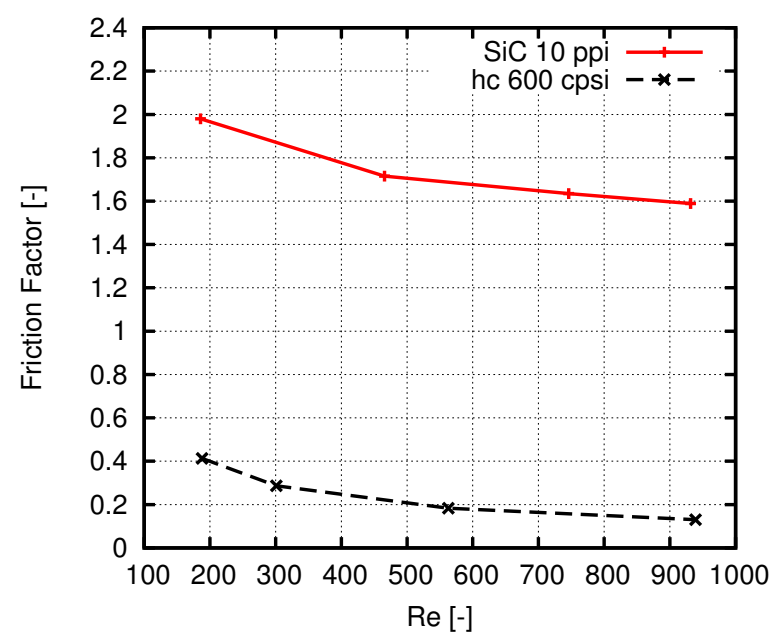

Figure 26: Friction factor versus Reynolds number for SiC-foam and honeycomb.

[9] F. Lucci, A. Della Torre, J. von Rickenbach, G. Montenegro, D. Poulikakos, and P. Dimopoulos Eggenschwiler. Performance of randomized kelvin cell structures as catalytic substrates: Mass-transfer based analysis. Chemical Engineering Science, 112:143-151, 2014.

[10] A. Della Torre, G. Montenegro, A. Onorati, and G. Tabor. CFD characterization of pressure drop and heat transfer inside porous substrates. Energy Procedia, 81:836 - 845, 2015. 69th Conference of the Italian Thermal Engineering Association, ATI 2014.

[11] L. Giani, G. Groppi, and E. Tronconi. Mass-transfer characterization of metallic foams as supports for structured catalysts. Industrial and Engineering Chemistry Research, 44(14):4993-5002, 2005.

[12] W. Xu, H. Zhang, Z. Yang, and J. Zhang. Numerical investigation on the flow characteristics and permeability of three-dimensional reticulated foam materials. Chemical Engineering Journal, 140(1-3):562-569, 2008.

[13] S. Mancin, C. Zilio, A. Cavallini, and L. Rossetto. Heat transfer during air flow in aluminum foams. International Journal of Heat and Mass Transfer, 53(21-22):4976-4984, 2010.

[14] F. Lucci, A. Della Torre, G. Montenegro, R. Kaufmann, and P. Dimopoulos Eggenschwiler. Comparison of geometrical, momentum and mass transfer characteristics of real foams to kelvin cell lattices for catalyst applications. International Journal of Heat and Mass Transfer, 108:341-350, 2017.

[15] Petros Maragos. Tutorial on advances in morphological image processing and analysis. Optical Engineering, 26(7):623-632, 1987. 


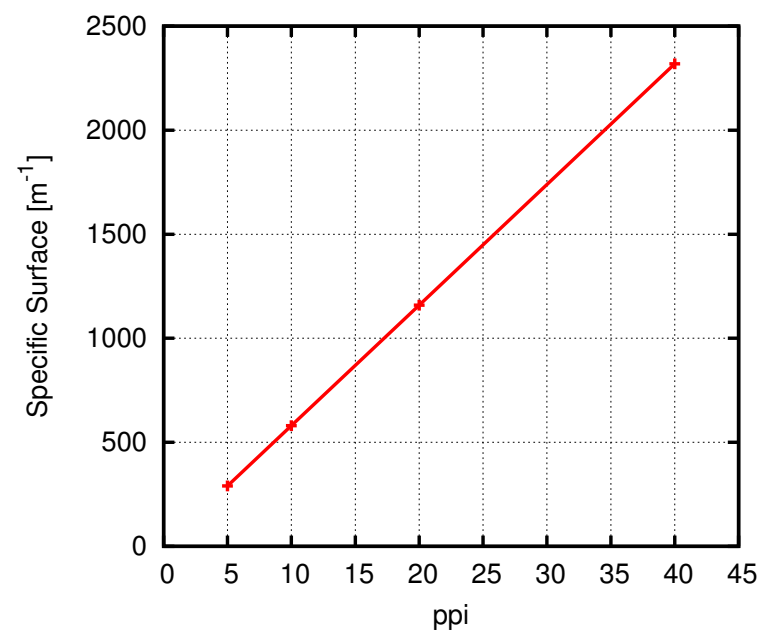

Figure 27: Specific surface $S_{V}$ vs pore density.

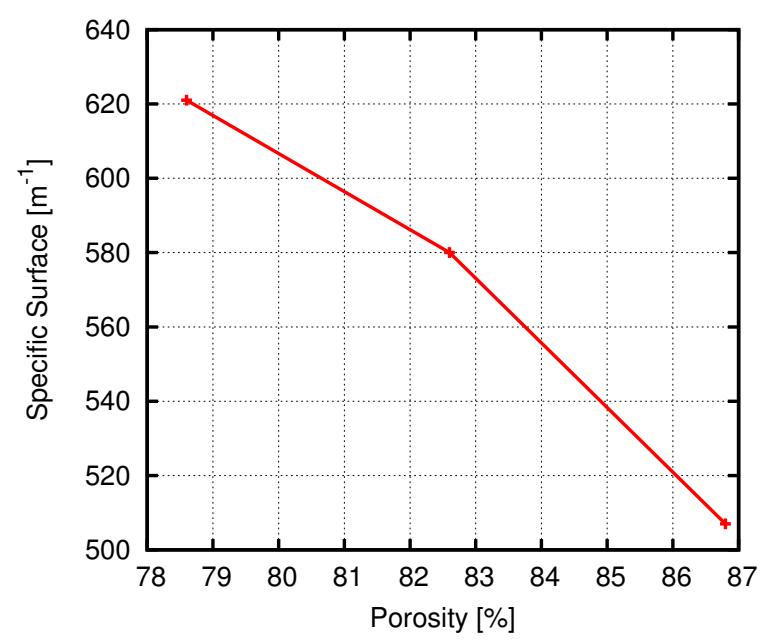

Figure 28: Specific surface $S_{V}$ vs porosity $\epsilon$.

[16] R.M. Haralick, S.R. Sternberg, and X. Zhuang. Image analysis using mathematical morphology. IEEE Transactions on Pattern Analysis and Machine Intelligence, PAMI-9(4):532-550, 1987.

[17] Z. Wu, C. Caliot, F. Bai, G. Flamant, Z. Wang, J. Zhang, and C. Tian. Experimental and numerical studies of the pressure drop in ceramic foams for volumetric solar receiver applications. Applied Energy, 87(2):504-513, 2010.

[18] P. Marsh, F. Acke, R. Konieczny, R. Bruck, and P. Hirth. Application guideline to define catalyst layout for maximum catalytic efficiency. SAE Technical Papers, 2001.

[19] A. Bhattacharya, V.V. Calmidi, and R.L. Mahajan. Thermophysical properties of high porosity metal foams. International Journal of Heat and Mass Transfer, 45(5):1017-1031, 2002. 